\title{
Existence Results for a Family of Equations of Fractional Resolvent
}

\author{
(Keputusan Kewujudan bagi Keluarga Persamaan Pecahan Berperingkat)
}

\author{
RABHA W. IBRAHIM*, SAYYEDAH A. QASEM \& ZAILAN SIRI
}

\section{ABSTRACT}

This study deals with the presence and distinction of bounded $m$-solutions (type mild) for a family of generalized integral and differential equations of spot order with fractional resolvent and indefinite delay.

Keywords: Fractional calculus; fractional differential equations; fractional differential operator

\section{ABSTRAK}

Kajian ini membincangkan kehadiran dan perbezaan terbatas m-penyelesaian (jenis lembut) untuk keluarga integral umum dan persamaan pembezaan titik tertib dengan pecahan berperingkat dan lengah tak tentu.

Kata kunci: Kalkulus pecahan; pecahan pembezaan pengoperasi; persamaan pembezaan pecahan

\section{INTRODUCTION}

Araya and Lizama (2008) imposed the concept of $\alpha$-resolvent families to establish the existence of m-solutions to the differential equation:

$$
D_{t}^{\alpha} u(t)=\Lambda u(t)+t^{n} \phi(t) \quad 1 \leq \alpha \leq 2, n \in Z,
$$

in a Banach space $\Xi$, for automorphism functions $\phi: R \rightarrow \Xi$. In addition, the authors prove the presence and distinction of:

$$
D_{t}^{\alpha} u(t)=\Lambda u(t)+\phi(t, u(t)), 1 \leq \alpha \leq 2,
$$

as well as:

$$
D_{t}^{\alpha} u(t)=\Lambda u(t)+\phi\left(t, u(t), u^{\prime}(t)\right), \quad 1 \leq \alpha \leq 2 .
$$

Numerous researchers established the existence and uniqueness of $\mathrm{m}$-solution for different types of fractional differential equations and differential-integral equations (Agarwal et al. 2012; Cuevas \& Lizama 2008; Diagana 2009; Ponce 2013). Furthermore, Cuevas and Lizama (2008) elected almost mild solutions for:

$$
D_{t}^{\alpha} u(t)=\Lambda u(t)+D_{t}^{\alpha-1} \phi(., u), 1<\alpha<2,
$$

where $\Lambda$ is a linear operator and $\phi(t, x)$ is almost automoorphic Lipschitz in $x$. Agarwal et al. (2012) studied analytic resolvent operator and existence results for fractional integro- differential equations of the form:

$$
D_{t}^{\alpha} u(t)=\Lambda u(t)+\int_{0}^{t} B(t-s) u(s) d s, \quad t>0, u(0)=u_{0} .
$$

Ponce (2013) considered the presence and distinction of bounded solutions for the linear fractional differential equation:

$$
D_{t}^{\alpha} u(t)=\Lambda u(t)+\int_{-\infty}^{t} a(t-s) \Lambda u(s) d s+\phi(t, u(t)), t \in \mathrm{R},
$$

where $\Lambda$ is a closed linear operator defined in a Banach space $\Xi, \alpha>0, a \in L^{1}\left(\mathrm{R}_{+}\right)$is a scalar-valued kernel and $f: \mathbf{R} \times \Xi \rightarrow \Xi$ substitutes some Lipschitz type conditions. Dhanapalan et al. (2014) created the presence and distinction of $\mathrm{m}$-solution of a class of nonlinear fractional integral-differential equations:

$$
\begin{aligned}
& D_{t}^{\alpha} u(t)+\Lambda u(t)+\int_{0}^{t} a(t-s) \Lambda u(s) d s+\int_{0}^{t} a(t-s) \psi(s, y(s)) d s \\
& +\varphi(t, u(t)), t \in[0, T], u(0)=u_{0},
\end{aligned}
$$

in a Banach space $\Xi$, where $0<\alpha<1$. The results are obtained by fixed point theorems. The results are established by using Krasnoselskii fixed point theorem and the contraction mapping principle. All the fractional operators are defined in the sense of the Riemann-Liouville fractional calculus.

In this conduct, we deal with the finding and regularity of $\mathrm{m}$ - solutions for a class of abstract fractional integraldifferential equations of the pattern:

$$
\begin{aligned}
& \mathrm{D}^{\alpha}\left(v(t)+\phi\left(t, v^{*}\right)\right)=\Lambda v(t)+\left(\int_{0}^{t} V_{\alpha}(t-\tau) v(\tau) d \tau\right)+ \\
& \psi\left(t, v^{*}\right), t \in J=[0, T], \\
& v_{0}=\varphi \in B,
\end{aligned}
$$

where $\Lambda: \operatorname{dom}(\Lambda) \subset \Xi \rightarrow \Xi$ and $V_{\alpha}(t): \operatorname{dom}(V(t)) \subset \Xi \rightarrow \Xi, t \geq$ 0 , are closed linear operators; $(\Xi,|| \mid)$ is a Banach space; the antiquity $v^{*}:(-\infty, 0] \rightarrow \Xi, v^{*}(\theta)=(t+\theta)$ accords in $B$ and $\phi, \psi: J \times B \rightarrow \Xi$ are devoted functions. Finally, $\mathrm{D}^{\alpha}$ stands the Caputo fractional derivative (Kilbas et al. 2006; Podlubny 1999), 


$$
\mathrm{D}^{\alpha} b(s)=\frac{1}{\Gamma(m-\alpha)} \int_{a}^{s} \frac{b^{(m)}(\delta)}{(s-\delta)^{\alpha+1-m}} d \delta,
$$

where $m$ is the closed integer greater than $\alpha$. Recently, different studies concerning the fractional differential and integral equations, such as Ulam stability and equations with maxima are included in the works of Agarwal et al. (2014), Ibrahim (2014), (2013), (2012a), (2012b) and Ibrahim and Jalab (2010).

\section{MATERIALS AND METHODS}

During the whole of this work, $(\Xi,||$.$) is B-space (the space$ of Banach) and $\Lambda: \operatorname{dom}(\Lambda) \subset \Xi \rightarrow \Xi, V(t) \subset \operatorname{dom}(V(t))$ $\subset \Xi \rightarrow \Xi, t \geq 0$, are closed linear operators applied on a common domain $\operatorname{dom}(\Lambda)$ which is dense in $\Xi$. To get our results, we suppose that the Cauchy problem:

$$
\mathrm{D}^{\alpha} v(t)=\Lambda v(t)+\int_{0}^{t} V_{\alpha}(t-\tau) v(\tau) d \tau, t \geq 0, v(0)=z \in \Xi,
$$

has an associated $\alpha$-resolvent bounded operator on $\Xi$.

Definition 2.1 Consider the family of bounded operators $\left(\mathrm{F}_{a}(t)_{t \geq 0}\right.$ from $\Xi$ into $\Xi$. Then it is called $\alpha$-resolvent operator family for system (3) if the following assumptions are satisfied:

$\mathrm{F}_{\alpha}(0)=i_{d}$, (the identity function); $\mathrm{F}_{\alpha}(). v \in ð([0, \infty): \Xi)$ for all $\mathrm{v} \in \Xi$;

$\Lambda \mathrm{F}_{\alpha}(). v \in ð([0, \infty): \Xi)$ and $\mathrm{F}_{a}(). v \in \mathcal{J}^{1}([0, \infty): v)$ for every $v \in \operatorname{dom}(\Lambda)$;

For each $v \in \operatorname{dom}(\Lambda)$ and $t \geq 0$,

$$
\begin{aligned}
& \mathrm{D}^{\alpha} \mathrm{F}_{\alpha}(t) v=\Lambda \mathrm{F}_{\alpha}(t) v+\int_{0}^{t} V_{\alpha}(t-\tau) \mathrm{F}_{\alpha}(\tau) v d \tau, \\
& \mathrm{D}^{\alpha} \mathrm{F}_{\alpha}(t) v=\mathrm{F}_{\alpha}(t) \Lambda \nu+\int_{0}^{t} \mathrm{~F}_{\alpha}(t-\tau) V_{\alpha}(\tau) \nu d \tau .
\end{aligned}
$$

Let $v \in ð(J ; \Xi)$ and assume the integral-differential Cauchy problem:

$$
\left\{\begin{array}{l}
\mathrm{D}^{\alpha} v(t)=\Lambda v(t)+\int_{0}^{t} V_{\alpha}(t-\tau) v(\tau) d \tau+v(t), t \in J, \\
v(0)=z \in \Xi .
\end{array}\right.
$$

We introduce the subsidiary concepts of m-solution and c-solution (classical) for framework (6):

Definition 2.2 A function $v \in \check{\partial}(J, \Xi)$ is called a m-solution of (6) on $J$, if $v(0)=z$ and

$$
v(t)=\mathrm{F}_{\alpha}(t) z+\int_{0}^{t} \mathrm{~F}_{\alpha}(t-\tau) v(\tau) d \tau, t \in J .
$$

Definition 2.3 A function $v \in ð(J, \Xi)$ is called a c-solution (classic solution) of (6) on $J$, if $v(0)=z$, $v(.) \in \varpi^{1}(J, \Xi) \cap ð(J:[\operatorname{dom}(\Lambda)])$ and $(6)$ is verified.

Let $B$ be a complete linear space of functions $\phi:(-\infty, 0] \rightarrow \Xi$, endowed with a semi-norm \|\|$_{B}$. We need the following assumptions in the sequel:

(A1) If $v:(-\infty, \varsigma+a) \rightarrow \Xi, a>0, \varsigma \in \mathrm{R}$, is continuous on $[\zeta, \zeta+a]$ and $v^{*}:(-\infty, \zeta+a) \rightarrow B$, then for every $t \in[\zeta, \zeta+a)$ we receive

$$
\begin{aligned}
& \|v(t)\| \leq H_{\alpha}\left\|v^{*}\right\|_{B} \cdot \\
& \left\|v^{*}\right\|_{B} \leq K_{\alpha}(t-\varsigma) \sup \{\|v(\tau)\|: \varsigma \leq \tau \leq t\}+M_{\alpha}(t-\varsigma)\|v(\varsigma)\|_{B},
\end{aligned}
$$

where $H_{\alpha}>0$ is a positive constant depending on $\alpha ; K_{\alpha}$, $M_{\alpha}:[0,8) \rightarrow[1, \infty), K_{\alpha}$ is continuous, $M_{\alpha}$ is locally bounded and $H_{\alpha}, K_{\alpha}, M_{\alpha}$ are independent of $v($.$) .$

(A2) The continuous function $v:(-\infty, \zeta+b] \rightarrow \Xi, b<T$ achieves $v_{\varsigma}^{*}=0$ and the derivative exists. If the function $w:(-\infty, 0] \rightarrow \Xi$ defined by $w(\theta)=0$ for $\theta<0$ and $w(0)=$ $v^{\prime}(\zeta) \in B$, then $\left\|\frac{1}{v} v^{*}-w\right\|_{B} \rightarrow 0, \quad v \rightarrow 0$.

Assume that $\left(\mathrm{U},\|\|_{u}\right)$ and $\left(\mathrm{V},\|\|_{v}\right)$ are Banach spaces. Here, the representation $L(\mathrm{U}, \mathrm{V})$ attitudes for the Banach space of bounded linear operators from $\mathrm{U}$ into $\mathrm{V}$ endowed with the uniform operator topology and we abridge this symbol to $L(\mathrm{~V})$ when $\mathrm{V}=\mathrm{U}$. The documentation, $B_{r}(v, \mathrm{~V})$ attitudes as the closed ball with center at $v$ and radius $r>$ 0 in V. In addition, for a bounded function $\gamma:[0, a] \rightarrow \mathrm{V}$ and $t \in[0, a], a<T$, we employ the realization:

$$
\|\gamma\|_{v, t}=\sup \left\{\|\gamma(\tau)\|_{v}: \tau \in[0, t]\right\}
$$

and the extension of this symbol to $\gamma^{*}$, when no turmoil regarding the space $\mathrm{V}$ appears.

Definition 2.4 Let $\Lambda: \Xi \rightarrow \Upsilon$ be a linear operator. The graph of $\Lambda$ is given by

$$
\operatorname{Gr}(\Lambda):=\{(s, \Lambda s) \in \Xi \times \Upsilon, s \in \operatorname{dom}(\Lambda)\}
$$

The graph norm is:

$$
\|s\|_{\Lambda}=\|s\|_{E}+\|\Lambda s\|_{\Upsilon} .
$$

In the supplement, $[\operatorname{dom}(\Lambda)]$ is the domain of $\Lambda$ accorded through the graph norm.

\section{Continuation of Mild Solutions}

In this section, we establish the presence of m-solutions of the fractional system (1)-(2). Motivated by (7), we introduce the coming notions of m-solutions: 
Definition 3.1 A function $v:(-\infty, b) \rightarrow \Xi, 0<b \leq T$ is demanded a m-solution (mild) of (1)-(2) on $J$, if $v_{0}=\phi ;\left.v\right|_{J} \in \delta(J: \Xi)$; the functions $\tau \rightarrow \Lambda \mathrm{F}_{\alpha}(t-\tau) \phi\left(\tau, v^{*}(\zeta)\right)$ and $\tau \rightarrow \int_{0}^{\tau} V_{\alpha}(\tau-\xi) \mathrm{F}_{\alpha}(t-\tau) \varphi\left(\xi, v^{*}(\xi)\right)$ are integrable on $J$ for every $t \in J$ and,

$$
\begin{aligned}
v(t)= & \mathrm{F}_{\alpha}(t)(\varphi+\phi(0, \varphi))-\phi\left(t, v_{t}^{*}\right)-\int_{0}^{t} \Lambda \mathrm{F}_{\alpha}(t-\tau) \phi\left(\tau, v_{\tau}^{*}\right) d \tau \\
& -\int_{0}^{t} \int_{0}^{\tau} V_{\alpha}(\tau-\xi) \mathrm{F}_{\alpha}(t-\tau) \phi\left(\xi, v_{\xi}^{*}\right) d \xi d \tau \\
& +\int_{0}^{t} \mathrm{~F}_{\alpha}(t-\tau) \psi\left(\tau, v_{\tau}^{*}\right) d \tau, t \in J,
\end{aligned}
$$

where $v^{*}(\xi):=v_{\xi}^{*}$.

It is well known that for all $\chi \in \Xi$ there exists a unique mild solution of the abstract Cauchy problem, if and only if, the resolvent set generated by $\Lambda$ is nonempty and for all $\chi \in \operatorname{dom}(\Lambda)$ there exists a unique classical solution of the Cauchy problem (Arendt et al. 2011; Theorem 3.1.12). Therefore, we utilize the resolvent family in all terms of $v(t)$ in Definition 3.1, to establish a unique classical solution for (1)-(2).

To prove our findings, we illustrate the following hypotheses:

$\left(\mathbf{C}_{1}\right)$ There exists a Banach space $\left(\Upsilon,\|\|_{\Upsilon}\right)$ continuously embedded in $\Xi$ such that,

$$
\forall t \in(0, a], \mathrm{F}_{\alpha}(t) \in L(\Xi, \Upsilon) \cap L(\Upsilon,[\operatorname{dom}(\Lambda)])
$$

and $\mathrm{V}_{\alpha}(t) \in L(\Upsilon, \Xi)$. Additionally, $\Lambda \mathrm{F}_{\alpha}(). v \in ð((0, a], \Xi)$ and $V_{\alpha}(). v \in \partial(J, \Xi)$ for every $v \in \Upsilon$.

Let,

$$
\left\|\Lambda \mathrm{F}_{\alpha}(t)\right\|_{\mathrm{L}(\Upsilon, \Xi)} \leq H_{1, \alpha}(t), \quad H_{1, \alpha}(.) \in L^{1}\left(J ; \mathrm{R}^{+}\right),
$$

and

$$
\begin{aligned}
& \left\|V_{\alpha}(\tau) \mathrm{F}_{\alpha}(t)\right\|_{\mathrm{L}(\Upsilon, \Xi)} \leq \mu_{\alpha}(\tau) H_{2, \alpha}(t), \\
& \left(0 \leq \tau<t \leq T, \quad\left(H_{2, \alpha}(.), \mu_{\alpha}(\tau)\right) \in L^{1}\left(J ; \mathrm{R}^{+}\right) \times L^{1}\left(J ; \mathrm{R}^{+}\right)\right) .
\end{aligned}
$$

$\left(\mathbf{C}_{2}\right)$ Let $\psi: J \times B \rightarrow \Xi$ be a function achieving the coming hypotheses:

$$
\psi(t, .): B \rightarrow \Xi \text { is continuous } \forall t \in J
$$

$\forall w \in \Xi$, the function $\psi(., w): J \rightarrow \Xi$ is strongly measurable;

Let $h_{\psi} \in \mathcal{\partial}(J ;[0, \infty))$ and the continuous nondecreasing function $\Omega_{\psi}:[0, \infty) \rightarrow(0, \infty)$ be such that $\|\psi(t, w)\| \leq h_{\psi}(t) \Omega_{\psi}\left(\mid w \|_{B}\right)$ for all $(t, w) \in J \times B$.

$\left(\mathbf{C}_{3}\right)$ The continuous function $\phi: J \times B \rightarrow \Xi$ is such that $\phi(J \times B) \subset \Upsilon$ and the constants $\kappa_{1}>0, \kappa_{2}>0$ accomplish $\|\phi(t, v)\|_{\Upsilon} \leq \kappa_{1}\|v\|_{B}+\kappa_{2}$ for each $(t, v) \in J \times B$.
$\left(\mathbf{C}_{4}\right)$ The functions $\phi: \mathrm{R} \times \mathrm{B} \rightarrow \Xi$ so that $\phi(J \times B) \subset \Upsilon$ and $\ell_{\phi}:[0, \infty) \rightarrow[0, \infty)$, are continuous carrying out

$$
\begin{aligned}
& \left\|\phi\left(t, v_{1}\right)-\phi\left(t, v_{2}\right)\right\|_{\curlyvee} \leq \ell_{\phi}(r)\left\|v_{1}-v_{2}\right\|_{B}, \\
& \left(t, v_{i}\right) \in J \times B_{r, a}(\varphi, B), i=1,2 .
\end{aligned}
$$

$\left(\mathbf{C}_{5}\right) \psi: \mathrm{R} \times \mathrm{B} \rightarrow \Xi$ and $\ell_{\psi}:[0, \infty) \rightarrow[0, \infty)$ are continuous executing:

$$
\begin{aligned}
& \left\|\psi\left(t, v_{1}\right)-\psi\left(t, v_{2}\right)\right\| \leq \ell_{\psi}(r)\left\|v_{1}-v_{2}\right\|_{B}, \\
& \left(t, v_{i}\right) \in J \times B_{r, \alpha}(\phi, B), i=1,2 .
\end{aligned}
$$

$\left(\mathbf{C}_{\varphi}\right)$ Let $0<b \leq T$ and $\mathrm{S}(b)=\left\{v:(-\infty, b] \rightarrow \Xi: v_{0}=0\right.$, $\left.v \mathrm{I}_{[0, b]} \in \partial([0, b]: \Xi)\right\}$ endowed with the uniform convergence topology on $[0, b]$. For each bounded set $\mathrm{B} \subset \mathrm{S}(b)$, the set of functions $\left\{t \rightarrow \phi\left(t, v_{t}^{*}+w_{t}^{*}\right): v, w \in \mathrm{B}\right\}$ is equicontinuous on $[0, b]$.

Remark 3.1 Condition $\left(\mathbf{C}_{\mathbf{1}}\right)$ implies the estimates:

$$
\begin{aligned}
& \left\|\Lambda \mathrm{F}_{\alpha}(t-\tau) v(\tau)\right\| \leq H_{1, \alpha}(t-\tau)\|v(\tau)\|_{\Upsilon}, \\
& \left\|V_{\alpha}(\tau-\zeta) \mathrm{F}_{\alpha}(t-\tau) v(\xi)\right\| \leq \mu_{\alpha}(\tau-\zeta) H_{2, \alpha}(t-\tau)\|v(\zeta)\|_{\Upsilon},
\end{aligned}
$$

we close out that the functions $\tau \rightarrow \Lambda \mathrm{F}_{\alpha}(t-\tau) v(\tau)$ and $\tau \rightarrow \int_{0}^{\tau} V_{\alpha}(\tau-\zeta) \mathrm{F}_{\alpha}(t-\zeta) v(\zeta) d \zeta$ are integrable on $[0, t]$.

Remark 3.2 In the remainder here, we let $\lambda:(-\infty, T] \rightarrow \Xi$ be so that

$$
\lambda(t)=\left\{\begin{array}{lc}
\phi(t), & t<0, \\
\mathrm{~F}_{\alpha}(t) \phi(0), & t \geq 0 .
\end{array}\right.
$$

Moreover,

$$
\begin{gathered}
M_{\alpha}=\sup _{s \in[0, b]} \mid \mathrm{F}_{\alpha}(s) \|_{L(X)}, K_{\alpha, b}=\sup _{s \in[0, b]} K_{\alpha}(s), \\
M_{\alpha, b}=\sup _{s \in[0, b]} M_{\alpha}(s), \ell_{\varphi, b}=\sup _{s \in[0, b]} \ell_{\varphi}(s), \ell_{\psi, b}=\sup _{s \in[0, b]} \ell_{\psi}(s) .
\end{gathered}
$$

Now, we may impose our first existence finding.

Theorem 3.1 Assume the hypotheses $\left(\mathbf{C}_{\mathbf{1}}\right)-\left(\mathbf{C}_{\mathbf{3}}\right)$ and $\left(\mathbf{C}_{\varphi}\right)$ are achieved. Suppose

$$
\begin{aligned}
& \kappa_{1} K_{\alpha}(0)\left\|i_{c}\right\|_{L(\Upsilon, \Xi)}<1, \mathrm{~F}_{\alpha}(.) \in \partial((0, T] ; \\
& L(\Xi ; \Upsilon)) \bigcap \partial((0, T] ; L(\Upsilon)), V_{\alpha}(.) \in L^{1}([0, T] \\
& L(\Upsilon, \Xi))
\end{aligned}
$$

with the following condition $\left(\mathbf{C}^{\prime}\right)$ : for each $t \in(0, T]$ and $r$ $>0$, there exist a compact set $C_{r}^{1}$ in Yand a compact set $C_{r}^{2}$ in $\Xi$ such that $\mathrm{F}_{\alpha}(t) \phi(\tau, v) \in C_{r}^{1}(t)$ and $\mathrm{F}_{\alpha}(t) \psi(\tau, v) \in C_{r}^{2}(t)$ for every $(\tau, v) \in[0, t] \times B_{r, a}(0, B)$. Then there exists at least one m-solution of (1)-(2) on $[0, b]$ for some $0<b \leq T$. 
Proof for $0<b \leq a$, we have the inequalities:

$$
\frac{K_{\alpha, a} M_{\alpha}}{\left(1-\mu_{\alpha}\right)} \int_{0}^{b} h_{\psi}(\tau) d \tau<\int_{C}^{\infty} \Omega_{\psi, \alpha}(\tau) d \tau,
$$

and

$$
\begin{gathered}
\mu_{\alpha}=\kappa_{1} K_{\alpha, b}\left(\left\|i_{c}\right\|_{L(\Upsilon, \Xi)}+\int_{0}^{b} H_{1, \alpha}(s) d s+\right. \\
\left.\int_{0}^{b} \mu_{\alpha}(\xi) d \xi \int_{0}^{b} H_{2, \alpha}(\tau) d \tau\right)<1,
\end{gathered}
$$

where,

$$
\begin{aligned}
C= & \frac{1}{1-\mu_{\alpha}}\left(\left(M_{\alpha, a}+K_{\alpha, a} M_{\alpha} H_{\alpha}\right)\|\phi\|_{B}+\right. \\
& \left.K_{\alpha, a} M_{\alpha}\|\varphi(0, \phi)\|+\frac{\kappa_{2} \mu_{\alpha}}{\kappa_{1}}\right) .
\end{aligned}
$$

Define a function space:

$$
\mathrm{S}(b)=\left\{v:(-\infty, b] \rightarrow \Xi: v_{0}=0,\left.v\right|_{[0, b]} \in C([0, b]: \Xi)\right\},
$$

endowed with the uniform convergence topology, we characterize the operator $\Psi: \mathrm{S}(b) \rightarrow \mathrm{S}(b)$ by $(\Psi v)(0)=0$ and

$$
\begin{aligned}
\Psi v(t)= & \mathrm{F}_{\alpha}(t) \varphi(0, \phi)-\varphi\left(t, v_{t}^{*}+w_{t}^{*}\right)-\int_{0}^{t} \Lambda \mathrm{F}_{\alpha}(t-\tau) \varphi\left(\tau, v_{t}^{*}+w_{t}^{*}\right) d \tau \\
& -\int_{0}^{t} \int_{0}^{\tau} V_{\alpha}(\tau-\xi) \mathrm{F}_{\alpha}(t-\tau) \varphi\left(\xi, v_{\xi}^{*}+w_{\xi}^{*}\right) d \xi d \tau \\
& +\int_{0}^{t} \mathrm{~F}_{\alpha}(t-\tau) \psi\left(\tau, v_{\tau}^{*}+w_{\tau}^{*}\right) d \tau, \quad t \in[0, b] .
\end{aligned}
$$

In view of Remark 3.1 and condition $\left(\mathbf{C}_{\mathbf{3}}\right)$ yield $\Psi \mathrm{S}(b)$ $\subset \mathrm{S}(b)$.

We proceed to establish that $\Psi$ satisfies the LeraySchauder Theorem. Let $\left(v^{n}\right)_{n \in \mathrm{N}}$ be a sequence in $\mathrm{S}(b)$ and $v \in \mathrm{S}(b)$ with $v^{n} \rightarrow v$ in $\mathrm{S}(b)$. Consequently, we retrieve $\mathrm{U}=\left\{v^{{ }^{*}}+w^{*}, v^{*}+w^{*}: \tau \in[0, b], n \in \mathrm{N}\right\}$ is relatively compact set in $B$ along with uniformly for as $n \rightarrow \infty$. Consequently, we receive that is uniformly on $[0, b]$ when $n \rightarrow \infty$ Hence the Lebesgue dominated convergence Theorem implies that $\Psi$ is continuous.

Next, we prove the boundedness of solutions of the integral equation $z=\lambda \Psi_{z}, \lambda \in(0,1)$. Let $v^{\lambda}$ be a solution of $z=\lambda \Psi_{z}, \lambda \in(0,1)$ and $v^{\lambda}$ be the function defined by:

$$
v^{\lambda}(s)=\left(M_{\alpha, b}+K_{b, \alpha} M H_{\alpha}\right)\|\phi\|_{B}+K_{\alpha, b} \sup _{\theta \in[0, s]}\left\|v^{\lambda}(\theta)\right\| .
$$

By employing $\left\|v_{s}^{\lambda}(t)\right\|_{B}+\left\|w_{s}\right\|_{B} \leq v^{\lambda}(s)$, we get that,

$$
\begin{aligned}
& \left\|v^{\lambda}(t)\right\| \\
\leq & M_{\alpha}\|\varphi(0, \phi)\|+\left\|i_{c}\right\|_{L(Y, \Xi)}\left(\kappa_{1} v^{\lambda}(t)+\kappa_{2}\right)+\int_{0}^{t} H_{1, \alpha}(t-s)\left(\kappa_{1} v^{\lambda}(s)+\kappa_{2}\right) d s \\
& +\int_{o}^{t} \int_{0}^{s} \mu_{\alpha}(s-\xi) H_{2, \alpha}(t-s)\left(\kappa_{1} v^{\lambda}(\xi)+\kappa_{2}\right) d \xi d s+M_{\alpha} \int_{0}^{t} h_{\psi}(s) \Omega_{\psi, \alpha}\left(v^{\lambda}(s)\right) d s \\
\leq & M_{\alpha}\|\varphi(0, \phi)\|+\kappa_{2}\left(\left\|i_{c}\right\|_{L(\Xi, Y)}+\int_{0}^{b} H_{1, \alpha}(s) d s+\int_{0}^{b} H_{2, \alpha}(s) d s+\int_{0}^{b} \mu_{\alpha}(\xi) d \zeta\right) \\
& +v^{\lambda}(t) \kappa_{2}\left(\left\|i_{c}\right\|_{L(\Xi, Y)}+\int_{0}^{b} H_{1, \alpha}(s) d s+\int_{0}^{b} H_{2, \alpha}(s) d s+\int_{0}^{b} \mu_{\alpha}(\xi) d \zeta\right) \\
& +M_{\alpha} \int_{0}^{t} h_{\psi}(s) \Omega_{\psi, \alpha}\left(v^{\lambda}(s)\right) d s
\end{aligned}
$$

which shows that,

$$
\begin{aligned}
v^{\lambda}(t) \leq & \left(M_{\alpha, b}+K_{b, \alpha} M_{\alpha} H_{\alpha}\right)\|\phi\|_{B}+K_{b, \alpha} M_{\alpha}\|\varphi(0, \phi)\| \\
& +\left(v^{\lambda}(t) \kappa_{1} K_{b, \alpha}+\kappa_{2} K_{\alpha, b}\right) \\
& \left.\left(\left\|i_{c}\right\|_{L(\Upsilon, \Xi)}+\int_{0}^{b} H_{1, \alpha}(s) d s+\int_{0}^{b} H_{2, \alpha}(s) d s+\int_{0}^{b} \mu_{\alpha}(\zeta) d \zeta\right)\right) \\
& +K_{b, \alpha} M_{\alpha} \int_{0}^{t} h_{\psi}(s) \Omega_{\psi, \alpha}\left(v^{\lambda}(s)\right) d s .
\end{aligned}
$$

Consequently, we obtain:

$$
v^{\lambda}(t) \leq C+\frac{K_{T, \alpha} M_{\alpha}}{\left(1-\mu_{\alpha}\right)} \int_{0}^{t} h_{\psi}(s) \Omega_{\psi, \alpha}\left(v^{\lambda}(s)\right) d s, t \in[0, b] .
$$

Indicated by:

$$
\hbar_{\lambda}(t):=C+\frac{K_{T, \alpha} M_{\alpha}}{\left(1-\mu_{\alpha}\right)} \int_{0}^{t} h_{\psi}(s) \Omega_{\psi, \alpha}\left(v^{\lambda}(s)\right) d s,
$$

we have

$$
\hbar_{\lambda}^{\prime}(t) \leq \frac{K_{T, \alpha} M_{\alpha}}{\left(1-\mu_{\alpha}\right)} h_{\psi}(t) \Omega_{\psi, \alpha}\left(\beta^{\lambda}(t)\right)
$$

and

$$
\int_{\hbar_{\lambda}(t)=c}^{\hbar_{\lambda}(t)} \frac{1}{\Omega_{\psi, \alpha}(s)} d s \leq \frac{K_{T, \alpha} M_{\alpha}}{\left(1-\mu_{\alpha}\right)} \int_{0}^{b} h_{\psi}(s)<\int_{c}^{\infty} \frac{1}{\Omega_{\psi, \alpha}(s)} d s .
$$

This inequality and (9) appearance that $\left\{\hbar^{\lambda}: \lambda \in(0,1)\right\}$ is uniformly bounded on $[0, b]$ which implies that $\left\{v^{\lambda}: \lambda \in(0,1)\right\}$ is bounded in the space $\mathrm{S}(b)$. To establish that $\Psi$ is completely continuous, we consider the atomization $\Psi=\Sigma_{i=1}^{3} \Psi_{i}$, where

$\Psi_{1} v(t)=\mathrm{F}_{\alpha} \varphi(0, \phi)-\varphi\left(t, v_{t}^{*}+w_{t}^{*}\right)-\int_{0}^{t} \Lambda \mathrm{F}_{\alpha}(t-s) \varphi\left(s, v_{s}^{*}+w_{s}^{*}\right) d s, \quad t \in I$,

$\Psi_{2} v(t)=\int_{0}^{t} \int_{0}^{s} V_{\alpha}(s-\zeta) \mathrm{F}_{\alpha}(t-s) \varphi\left(\zeta, v_{\zeta}^{*}+w_{\zeta}^{*}\right) d \zeta d s, t \in I$,

$\Psi_{3} v(t)=\int_{0}^{t} \mathrm{~F}_{\alpha}(t-s) \psi\left(s, v_{s}^{*}+w_{s}^{*}\right) d s$,

where $(\Psi x)_{0}=0$ for $i=1,2,3$. We shall use the notations $B_{\rho}=B_{\rho}\left(0, \mathrm{~S}(b), \rho>0\right.$ and $\rho^{*}=\left(M_{\alpha, T}+K_{\alpha, T} M_{\alpha} H_{\alpha}\right)\|\varphi\|_{B}+K_{\alpha} \rho$.

Step 1. Our aim was to prove $\left(\Psi_{2} B_{\rho, \alpha}\right)(t)=\left\{\Psi_{2} v(t): v \in B_{\rho}\right.$, $\forall t \in[0, b]\}$ is relatively compact in $\Xi$.

The trivial case, when $t=0$. Therefore, we put $0<\varepsilon<t<b$. From the hypotheses, we may consider the inequality:

$$
0=t_{0}<t_{1}<\ldots t_{n}=t-\varepsilon
$$

such that $\left\|\mathrm{F}_{\alpha}(t-s)-\mathrm{F}_{\alpha}\left(t-s^{\prime}\right)\right\|_{L(Y)} \leq \varepsilon$ for every $s, s^{\prime} \in\left[t_{i}, t_{i+1}\right]$, $i=0,1,2, \ldots, n-1$. Let $v \in B_{\rho}$. In virtue of the mean value theorem, we impose:

$$
\begin{aligned}
\Psi_{2} v(t)= & \sum_{i=1}^{n} \int_{t_{i-1}}^{t_{i}} \int_{0}^{s} V_{\alpha}(s-\zeta) \mathrm{F}_{\alpha}\left(t-t_{i}\right) \varphi\left(\zeta, v_{\zeta}^{*}+w_{\zeta}^{*}\right) d \zeta d s \\
& +\sum_{i=1}^{n} \int_{t_{i-1}}^{t_{i}} \int_{0}^{s} V_{\alpha}(s-\zeta)\left(\mathrm{F}_{\alpha}(t-s)-\mathrm{F}_{\alpha}\left(t-t_{i}\right)\right) \varphi\left(\zeta, v_{\zeta}^{*}+w_{\zeta}^{*}\right) d \zeta d s \\
& +\int_{t_{n}}^{t} \int_{0}^{s} V_{\alpha}(s-\zeta) \mathrm{F}_{\alpha}\left(t-t_{i}\right) \varphi\left(\zeta, v_{\zeta}^{*}+w_{\zeta}^{*}\right) d \zeta d s
\end{aligned}
$$


which shows that is relatively compact in $\Xi$.

Step 2. The set $\Psi_{2} B \rho=\left\{\Psi_{2} v: v \in B_{\rho}\right\}$ is equicontinuous on $[0, b]$.

Suppose $0<\varepsilon<t<b$ and $0<\delta<\varepsilon$ such that $\left\|\mathrm{F}_{\alpha}(s)-\mathrm{F}_{\alpha}\left(s^{\prime}\right)\right\|_{L(Y)} \leq \varepsilon$ for all $s, s^{\prime} \in[\varepsilon, b]$ with $\left|s-s^{\prime}\right| \leq \delta$. In view of the above assumption, for $v \in B_{\rho}$ and $0<\hbar^{*}<T$ with $t+\hbar^{*} \in[0, b]$, implies that:

$$
\left\|\Psi_{2} v\left(t+\hbar^{*}\right)-\Psi_{2} v(t)\right\|
$$

which shows that $\Psi_{2} B_{\rho}$ equicontinuity at $t \in(0, T)$. Hence, $\Psi_{2}$ is completely continuous. By utilizing (Hernandez \& McKibben 2005; Lemma 3.1], yields $\Psi_{1}$ and $\Psi_{3}$ are completely continuous. In view of Leray Schauder Theorem, we come to conclude that $\Psi$ has a fixed point $v$ $\in \mathrm{S}(b)$. Apparently, $u=v+w$ represents as a m-solution of in the interval $[0, b]$. This completes the proof.

By employing the records occurred in Remark 3.2, we get the following theorem:

Theorem 3.2 Let the status and $\left(\mathbf{C}_{\mathbf{1}}\right),\left(\mathbf{C}_{\mathbf{4}}\right)$ and $\left(\mathbf{C}_{\mathbf{5}}\right)$ be hold. $K(0) \ell_{\varphi}(0)\left\|i_{c}\right\|_{L(Y, \Xi)}<1$, If then there exists a unique m-solution of $(1)-(2)$ in $J$.

Proof: Let $b_{1}, r, C_{\phi}, C_{\psi}$ be positive constants satisfying $\|\phi(t, w)\|_{\Upsilon} \leq C_{\phi},\|\psi(t, w)\| \leq C_{\psi}$ where $(t, w) \in\left[0, b_{1}\right] \times B_{r}(\varphi, B)$. Now, we apply $0<b<b_{1}$ and $0<q<b$ in

$$
\begin{gathered}
\mu_{\alpha}=\ell_{\varphi, b}\left\|i_{c}\right\|_{L}(\Upsilon, \Xi) K_{b}<1, q K_{b, \alpha}+\sup _{\theta \in[0, b]}\left\|w_{\theta}-\phi\right\|_{B}<r, \\
\left\|\mathrm{~F}_{\alpha}(\theta) \varphi\left(\theta, w_{\theta}\right)\right\|_{b}+M_{\alpha}\left\|\varphi(0, \phi)-\varphi\left(\theta, w_{\theta}\right)\right\|_{b} \leq \frac{\left(1-\mu_{\alpha}\right) q}{3}, \\
C_{\varphi} \int_{0}^{b} H_{1, \alpha}(s) d s+C_{\varphi} \int_{0}^{b} H_{2, \alpha}(\xi) d \xi \int_{0}^{b} \mu_{\alpha}(s) d s+b M_{\alpha} C_{\psi} \leq \frac{\left(1-\mu_{\alpha}\right) q}{3}, \\
K_{b, \alpha}\left[\ell_{\varphi, b} \int_{0}^{b} H_{1, \alpha}(s) d s+\ell_{\varphi, b} \int_{0}^{b} H_{2, \alpha}(\xi) d \xi \int_{0}^{b} \mu_{\alpha}(s) d s+b M_{\alpha} \ell_{\psi, b}\right] \\
<\frac{\left(1-\mu_{\alpha}\right) q}{3} .
\end{gathered}
$$

Let $\Psi$ as in the proof of Theorem 3.1. Our target is to show that $\Psi$ is a contraction mapping from $B_{q}(0, \mathrm{~S}(b))$ into $B_{q}(0, \mathrm{~S}(b))$. For $v \in \mathrm{S}(b)$ we impose:

$$
\left\|v_{t}^{*}+w_{t}^{*}-\phi\right\|_{B} \leq\left\|v_{t}^{*}\right\|_{B} \leq K_{b, \alpha} q+\sup _{t \in[0, b]}\left\|w_{t}^{*}-\phi\right\|_{B}<r .
$$

Hence,

$$
\begin{aligned}
& \|\Psi v(t)\| \\
\leq & \left\|\mathrm{F}_{\alpha}(t)\left(\varphi(0, \phi)-\varphi\left(t, w_{t}^{*}\right)\right)\right\|+\left\|\mathrm{F}_{\alpha}(t)\left(\varphi\left(t, w_{t}^{*}\right)-\varphi\left(t, w_{t}^{*}\right)\right)\right\| \\
& +\left\|\left(\varphi\left(t, w_{t}^{*}\right)-\varphi\left(t, v_{t}^{*}-w_{t}^{*}\right)\right)\right\|+\int_{0}^{t}\left\|\Lambda \mathrm{F}_{\alpha}(t-s) \varphi\left(s, v_{s}^{*}+w_{s}^{*}\right)\right\| d s \\
& \int_{0}^{t} \int_{0}^{s} V_{\alpha}(s-\xi) \mathrm{F}_{\alpha}(t-s) \varphi\left(\xi, v_{\zeta}^{*}+w_{\xi}^{*}\right) d \zeta d s+M_{\alpha} C_{\psi} b \\
\leq \quad & \left\|\varphi(0, \phi)-\varphi\left(\theta, w_{\theta}^{*}\right)\right\|_{B}+\left\|\left(\mathrm{F}_{\alpha}(\theta)\right) \varphi\left(\theta, w_{\theta}^{*}\right)\right\|_{B}+\left\|i_{c}\right\|_{L(Y, \xi)} \ell_{\varphi, b}\left\|v_{t}^{*}\right\|_{B} \\
& +C_{\varphi} \int_{0}^{b} H_{1, \alpha}(s) d s+C_{\varphi} \int_{0}^{b} H_{2, \alpha}(\gamma) d \gamma \int_{0}^{b} \mu_{\alpha}(\xi) d \zeta+M_{\alpha} C_{\psi} b \\
\leq & \left(1-\mu_{\alpha}\right) q+\left\|i_{c}\right\|_{L(Y, \Xi)} \ell_{\varphi, b} K_{b, \alpha}\|v\|_{B},
\end{aligned}
$$

which admits $\Psi_{v} \in B_{q}(0, \mathrm{~S}(b))$. However, for $v, u \in \mathrm{S}(b)$ and $t \in J$, we have:

$$
\begin{aligned}
& \|\Psi u(t)-\Psi v(t)\| \\
\leq & \left\|i_{c}\right\|_{L(Y, \Xi)} \ell_{\varphi, b} K_{b, \alpha}\|u-v\|_{b}+\ell_{\varphi, b} K_{b, \alpha} \int_{0}^{b} H_{1, \alpha}(t-s) d s\|u-v\|_{\mathrm{B}} \\
& +\left(\ell_{\varphi, b} K_{b, \alpha} \int_{0}^{t} \int_{0}^{s} \mu_{\alpha}(s-\xi) H_{2, \alpha}(t-s) d \xi d s+M_{\alpha} \ell_{\psi, b} K_{b, \alpha} T\right)\|u-v\|_{\mathrm{B}} \\
\leq & \left(\left\|i_{c}\right\|_{L(Y, \Xi)} \ell_{\varphi, b} K_{b, \alpha}+\frac{1-\mu_{\alpha}}{3}\right)\|u-v\|_{\mathrm{B}},
\end{aligned}
$$

this yields $\Psi$ is a contraction on $B_{q}(0, \mathrm{~S}(b))$.

Theorem 3.3 Let suppositions $\left(\mathbf{C}_{\mathbf{1}}\right),\left(\mathbf{C}_{\mathbf{4}}\right)$ and $\left(\mathbf{C}^{\prime}\right)$ be hold. Assume, Then (1)-(2) admits a unique m-solution.

Proof: Let $b, r, q$ and $\Psi$ as in the proof of Theorem 3.2 and let $\Psi=\Psi_{1}=\Psi_{2}$ where $\left(\Psi_{i} v\right)_{0}=0$ such that:

$$
\begin{aligned}
\Psi_{1} v(t)= & \mathrm{F}_{\alpha}(t) \varphi(0, \phi)-\varphi\left(t, v_{t}^{*}+w_{t}^{*}\right) \\
& -\int_{0}^{t} \Lambda \mathrm{F}_{\alpha}(t-s) \varphi\left(s, v_{s}^{*}+w_{s}^{*}\right) d s \\
& +\int_{0}^{t} \int_{0}^{s} V_{\alpha}(s-\xi) \mathrm{F}_{\alpha}(t-s) \varphi\left(\xi, v_{\xi}^{*}+w_{\xi}^{*}\right) d \xi d s, \\
\Psi_{2} v(t)= & \int_{0}^{t} \mathrm{~F}_{\alpha}(t-s)\left(s, v_{s}^{*}+w_{s}^{*}\right) d s, \quad t \in[0, b] .
\end{aligned}
$$

By Theorem 3.2, we receive $\Psi\left(B_{q}(0, \mathrm{~S}(b))\right) \subset B_{q}(0, \mathrm{~S}(b))$ and that $\Psi_{1}$ is completely continuous on $B_{q}(0, \mathrm{~S}(b))$. Furthermore, in the same manner of Theorem 3.1, it follows that $\Psi_{2}$ is completely continuous on $B_{q}(0, \mathrm{~S}(b))$, which concludes $\Psi$ is a condensing operator (Martin 1987; Theorem 4.3.2).

\section{DISCUSSION}

Here, we introduce utilization of our abstract outcomes. We start with $\Xi$ is finite dimensional. Consider the following neutral fractional differential equation:

$$
\begin{aligned}
& \mathrm{D}^{\alpha}\left(v(t)+\varphi\left(t, v^{*}\right)\right)=\Lambda v(t)+\psi\left(t, v^{*}\right), \\
& t \in J=[0, a], v_{0}=\phi \in B .
\end{aligned}
$$

Suppose that $\Lambda$ is a generator of the bounded linear operators $\left(\mathrm{T}_{\alpha}(t)_{t \geq 0}\right.$ on $\Xi$ achieving $\left\|T_{\alpha}(t)\right\| \leq M_{\alpha}, M_{\alpha}>0$ for every $t \in[0, \alpha]$. We define fractional power linear operator $(-\Lambda)^{\alpha}$ for $0<\alpha \leq 1$, with its domain $\operatorname{dom}(-\Lambda)^{\alpha}$. In addition, $\operatorname{dom}(-\Lambda)^{\alpha}$ is dense in $\Xi$ such that:

$$
\|v\|_{\alpha}=\left\|(-\Lambda)^{\alpha} v\right\|
$$

define a norm on $\operatorname{dom}(-\Lambda)^{\alpha}$. If $\left(\Xi_{\alpha}, \alpha \in(0,1)\right.$ denotes the space $\operatorname{dom}(-\Lambda)^{\alpha}$ equipped with the norm \|\|$_{\alpha}$, then $\left(\Xi_{\alpha},\|\|_{\alpha}\right)$ is a Banach space continuously embedded in $\Xi$ and there occurs $C_{\alpha}>0$ satisfying $\left\|(-\Lambda)^{\alpha} \mathrm{T}_{\alpha}(t)\right\| \leq C_{\alpha} t^{-\alpha}$ for $t>0$.

The following outcomes are special cases of Theorems 3.1, 3.2 and 3.3. We discuss the case, $\mathrm{F}_{\alpha}(t)=\mathrm{T}_{\alpha}(t)$ for $t \geq 0$.

Theorem 4.1 Suppose the facts $\left(\mathbf{C}_{2}\right),\left(\mathbf{C}_{3}\right)$ and $\left(\mathbf{C}_{\varphi}\right)$ are fulfilled with $\Upsilon=\Xi_{\alpha}, \alpha \in(0,1),\left(\mathrm{T}_{\alpha}(t)_{t \geq 0}\right.$ is compact and 
$K(0)\left\|i_{c}\right\|_{L\left(\Xi_{\alpha}, \Xi\right)} c_{1}<1$. Then there occurs a m-solution of (11) on $[0, a]$.

Theorem 4.2 Assume that the status $\left(\mathbf{C}_{\mathbf{4}}\right)$ and $\left(\mathbf{C}_{5}\right)$ are contented with $\Upsilon=\Xi_{\alpha}$, for some $\alpha \in(0,1)$. If $K(0)\left\|i_{c}\right\|_{L\left(\Xi_{\alpha}, \Xi\right)} \ell_{\varphi}(0)<1$, then there arises a unique m-solution of $(11)$ on $[0, a]$.

Theorem 4.3 Consider the condition $\left(\mathbf{C}_{\mathbf{4}}\right)$ is verified with $\Upsilon=\Xi_{\alpha}$, for some $\alpha \in(0,1)$. Assume that the semigroup $\left(\mathrm{T}_{\alpha}(t)_{t \geq 0}\right.$ is compact and $K(0) \ell_{\varphi}(0)\left\|i_{c}\right\|_{L\left(\Xi_{\alpha}, \Xi\right)}<1$. Then there derives a m- solution of (11) on $[0, a]$.

Example 4.4 Consider the equation:

$$
\mathrm{D}^{\alpha}\left(v(t)+\frac{1}{2} v^{*}(t)\right)=v(t)+\frac{1}{2} v^{*}(t), t \in J=[0, a], v_{0}=\phi \in B .
$$

It is clear that $\phi=\psi$ satisfied conditions $\left(\mathbf{C}_{4}\right)$ and $\left(\mathbf{C}_{5}\right)$ with a constant $\ell_{\varphi}=\ell_{\psi}=\frac{1}{2}$. Moreover, $\left\|v^{*}\right\|_{B} \leq 1$, where $B:=C[0,1]$; thus $K=1$ and consequently $K(0) \ell_{\varphi}(0)\left\|i_{c}\right\|_{L\left(\Xi_{\alpha}, \Xi\right)}=\frac{1}{2}<1$. Hence in view of Theorem 4.2, we conclude that (12) has a unique m-solution.

\section{CONCLUSION}

We have shown that there exists a unique mild solution of the abstract Cauchy problem, if and only if, there exists a unique classical solution of the Cauchy. Therefore, we have used the fractional resolvent family in all terms of (1)-(2) to establish the unique classical solution.

\section{REFERENCES}

Agarwal, R.P., Ahmad, B., Alsaedi, A. \& Al-Hutami, H. 2014. On nonlinear fractional q-difference equations involving two fractional orders with three-point non-local boundary conditions. Dyn. Contin. Discrete Im- puls. Syst. Ser. A Math. Anal. 21: 135-151.

Agarwal, R.P., dos Santos, J.P. \& Cuevas, C. 2012. Analytic resolvent operator and existence results for fractional integrodifferential equations. Journal of Abstract Differential Equations and Applications 2(2): 26-47.

Araya, D. \& Lizama, C. 2008. Almost automorphic mild solutions to fractional differential equations. Nonlinear Anal. 69: 3692-3705.

Arendt, W., Batty, C.J., Hieber, M. \& Neubrander, F. 2011. Vectorvalued Laplace Transforms and Cauchy Problems. 2nd ed. Berlin: Birkhäuser, Springer.
Cuevas, C. \& Lizama, C. 2008. Almost automorphic solutions to a class of semi linear fractional differential equations. Appl. Math. Lett. 21: 1315-1319.

Dhanapalan, V., Thamilselvan, M. \& Chandrasekaran, M. 2014. Existence and uniqueness of mild solutions for fractional integrodifferential equations. Appl. Comput Math-Bak.3(1): 32-37.

Diagana, T. 2009. Existence of solutions to some classes of partial fractional differential equations. Nonlinear Anal. 71: 5269-5300.

Hernandez, E. \& McKibben, M. 2005. Some comments on: Existence of solutions of abstract nonlinear second order neutral functional integro-differential equations. Comput. Math. Appl. 50: 655-669.

Ibrahim, R.W. 2014. Solutions to systems of arbitrary -order differential equations in complex domains. Electronic Journal of Differential Equations 46: 1-13.

Ibrahim, R.W. 2013. Modified fractional Cauchy problem in a complex domain. Advances in Difference Equations 149: $1-10$.

Ibrahim, R.W. 2012a. Complex transforms for system of fractional differential equations. Abstract and Applied Analysis Artical number 814759.

Ibrahim, R.W. 2012b. Ulam stability for fractional differential equation in complex domain. Abstract and Applied Analysis Artical number 649517.

Ibrahim, R.W. \& Jalab, H.A. 2010. Existence of the solution of fractional integral inclusion with time delay. Miskolic Mathematical Journal 2(11): 139-150.

Kilbas, A.A., Srivastava, H.M. \& Trujillo, J.J. 2006. Theory and Applications of Fractional Differential Equations. Vol. 204 (North-Holland, Mathematics Studies). Elsevier Science.

Martin, R.H. 1987. Nonlinear Operators and Differential Equations in Banach Spaces. Florida: Robert E. Krieger Publ. Co.

Podlubny, I. 1999. Fractional Differential Equations. London: Acad. Press.

Ponce, R. 2013. Bounded mild solutions to fractional integrodifferential equations in Banach spaces. Semigroup Forum 87: 377-392.

Institute of Mathematical Sciences

University Malaya

50603 Kuala Lumpur

Malaysia

*Corresponding author; email: rabhaibrahim@yahoo.com

Received: 4 June 2014

Accepted: 25 July 2014 